\title{
ON AN INVERSE PROBLEM FOR A PARABOLIC EQUATION IN A DOMAIN WITH MOVING BOUNDARIES
}

\author{
ADALAT YA. AKHUNDOV AND ARASTA SH. HABIBOVA
}

\begin{abstract}
This paper considers the inverse problem of determining the unknown coefficient on the right-hand side of a parabolic equation in a domain with moving boundaries. An additional condition for finding the unknown coefficient, which depends on the variable time, is given in integral form. A theorem on uniqueness and "conditional" stability of the solution is proved.
\end{abstract}

\section{Introduction and statement of the problem}

In the theoretical study of energy or mass transfer processes associated with a change in the aggregate state of substance, in the theory of brittle fracture, in the study of growth of crack surfaces, in filtration problems, etc., there are problems in domains with moving boundaries (see, e.g., [3-6]).

From a mathematical point of view, boundary-value transfer problems in domains with moving boundaries are fundamentally different from classical problems.

In this paper, we consider the inverse problem of determining the unknown coefficient on the right-hand side of the parabolic equation. The inverse problem is studied in a domain with movable boundaries and with additional integral condition. The sought coefficient depends on the time variable.

Let $\gamma_{1}(t), \gamma_{2}(t) t \in[0, T], 0<T=$ const be the given functions, $(x, t)$ be an arbitrary point in the bounded domain $D=\left(\gamma_{1}(t), \gamma_{2}(t)\right) \times(0, T],[a, b]$ be the projection of the domain $D$ into the axis $O X$. The spaces $C^{l}(\cdot), C^{l+\alpha}(\cdot), C^{l, l / 2}(\cdot)$, $C^{l+\alpha,(l+\alpha) / 2}(\cdot), l=0,1,2, \quad 0<\alpha<1$ and the norms in these spaces are defined as in $[5$, Chapter 1$]$ :

$$
\|p\|_{D}^{(l)}=\sum_{k=0}^{l} \sup _{D}\left|\frac{\partial^{k} p(x, t)}{\partial x^{k}}\right|,\|q\|_{T}^{(l)}=\sum_{k=0}^{l} \sup _{[0, T]}\left|\frac{d^{k} q(t)}{d t^{k}}\right| .
$$

We consider the following inverse problem on determining a pair of functions $\{f(t), u(x, t)\}$ :

$$
\begin{gathered}
u_{t}-u_{x x}=f(t) g(x) \quad(x, t) \in D, \\
u(x, 0)=\varphi(x) \quad x \in\left[\gamma_{1}(0), \gamma_{2}(0)\right],
\end{gathered}
$$

2010 Mathematics Subject Classification. 35K40, 35K57.

Key words and phrases. Inverse problem, equation of parabolic type, uniqueness, "conditional" stability, domains with moving boundaries. 


$$
\begin{gathered}
u\left(\gamma_{1}(t), t\right)=\psi_{1}(t), \quad u\left(\gamma_{2}(t), t\right)=\psi_{2}(t), t \in[0, T], \\
\int_{\gamma_{1}(t)}^{\gamma_{2}(t)} u(x, t) d x=h(t), \quad t \in[0, T]
\end{gathered}
$$

where $g(x), \varphi(x), \psi_{1}(t), \psi_{2}(t), h(t)$ are the given functions, $u_{t}=\frac{\partial u}{\partial t}, u_{x}=\frac{\partial u}{\partial x}$, $u_{x x}=\frac{\partial^{2} u}{\partial x^{2}}$.

The coefficient inverse problem for a parabolic equation in domains with moving boundaries were studied in the papers $[1,2]$.

Problem (1.1)-(1.4) belongs to the class of Hadamard ill-posed problems. Therefore, this problem should be treated proceeding from the general concepts of the theory of ill-posed problems.

We make the following assumptions for the data of problem (1.1)-(1.4):

$$
\begin{aligned}
& 1^{0} . g(x) \in C^{\alpha}[a, b], \int_{\gamma_{1}(t)}^{\gamma_{2}(t)}|g(x)| d x \geq c_{1}>0 ; \\
& 2^{0} \cdot \varphi(x) \in C^{2+\alpha}\left[\gamma_{1}(0), \gamma_{2}(0)\right] ; \\
& 3^{0} \cdot \psi_{1}(t), \psi_{2}(t) \in C^{1+\alpha}[0, T], \varphi\left(\gamma_{1}(0)\right)=\psi_{1}(0), \varphi\left(\gamma_{2}(0)\right)=\psi_{2}(0) ; \\
& 4^{0} \cdot h(t) \in C^{1+\alpha}[0, T] ; \\
& 5^{0} \cdot \gamma_{1}(t), \gamma_{2}(t) \in C^{1+\alpha}[0, T], 0<c_{2} \leq \gamma_{2}(t)-\gamma_{1}(t) \leq c_{3}<+\infty, t \in[0, T] \\
& \text { where } c_{1}, c_{2} \text { and } c_{3} \text { are positive constants, } \alpha \in(0,1) .
\end{aligned}
$$

Definition 1.1. The pair of functions $\{f(t), u(x, t)\}$ is called the solution of problem (1.1)-(1.4) if

1) $f(t) \in C[0, T]$

2) $u(x, t) \in C^{2,1}(D) \bigcap C(\bar{D})$;

3) The conditions (1.1)-(1.4) hold for these functions.

It can be shown that if $\{f(t), u(x, t)\}$ is a solution to problem (1.1)-(1.4) in the sense of definition 1.1, then these functions will satisfy relations (1.1), (1.2), (1.3) and

$$
\begin{gathered}
f(t)=\left[h_{t}(t)-\psi_{2}(t) \gamma_{2 t}(t)+\psi_{1}(t) \gamma_{1 t}(t)-u_{x}\left(\gamma_{2}(t), t\right)+u_{x}\left(\gamma_{1}(t), t\right)\right] / \\
/ \int_{\gamma_{1}(t)}^{\gamma_{2}(t)} g(x) d x, \quad t \in[0, T] .
\end{gathered}
$$

Formula (1.5) is obtained by integrating equation (1.1) through the interval $\left(\gamma_{1}(t), \gamma_{2}(t)\right)$ with respect to the variable $x$, taking into account the assumptions $1^{0}-5^{0}$.

Let us call problem (1.1), (1.2), (1.3) and (1.5) problem $A$.

\section{On the uniqueness and "conditional" stability of a solution}

The uniqueness theorem and the estimate of stability for the solutions of inverse problems occupy a central place in investigation of their well-posedness.

Define the following set:

$$
\begin{gathered}
K_{\alpha}=\left\{(f, u)\left|f(t) \in C^{\alpha}[0, T],\right| f(t) \mid \leq c_{4}, t \in[0, T], u(x, t) \in C^{2+\alpha, 1+\alpha / 2}(\bar{D}),\right. \\
\left.|u|,\left|u_{x}\right|,\left|u_{x x}\right| \leq c_{5}, \quad(x, t) \in \bar{D}\right\},
\end{gathered}
$$


where $c_{4}, c_{5}$ are some positive constants. Let us assume that the two input sets $\left\{g(\cdot), \varphi(\cdot), \psi_{1}(\cdot), \psi_{2}(\cdot), h(\cdot)\right\}$ and $\left\{\bar{g}(\cdot), \bar{\varphi}(\cdot), \bar{\psi}_{1}(\cdot), \bar{\psi}_{2}(\cdot), \bar{h}(\cdot)\right\}$ are given for Problem $A$. For brevity of the further exposition, Problem $A$ with the second input set we will call Problem $\bar{A}$. Let $\{f(t), u(x, t)\}$ and $\{\bar{f}(t), \bar{u}(x, t)\}$ be solutions of problems $A$ and $\bar{A}$, respectively.

Theorem 2.1. Let the following conditions hold

1) The functions $\left\{g(\cdot), \varphi(\cdot), \psi_{1}(\cdot), \psi_{2}(\cdot), h(\cdot)\right\}$ and $\left\{\bar{g}(\cdot), \bar{\varphi}(\cdot), \bar{\psi}_{1}(\cdot), \bar{\psi}_{2}(\cdot), \bar{h}(\cdot)\right\}$ satisfy conditions $1^{0}-4^{0}$, respectively;

2) The functions $\gamma_{1}(t), \gamma_{2}(t)$ satisfy condition $5^{0}$;

3) Solutions of problems $A$ and $\bar{A}$ exist in the sense of definition 1.1 and they belong to the set $K_{\alpha}$.

Then there exists a $T^{*}\left(0<T^{*} \leq T\right)$, such that for $(x, t) \in\left[\gamma_{1}(t), \gamma_{2}(t)\right] \times\left[0, T^{*}\right]$ the solution of problem (1.1)-(1.4) is unique, and the stability estimate

$$
\begin{gathered}
\|u-\bar{u}\|_{D}^{(0)}+\|f-\bar{f}\|_{T}^{(0)} \leq c_{6}\left[\|g-\bar{g}\|_{[a, b]}^{(0)}+\right. \\
\left.+\|\varphi-\bar{\varphi}\|_{[a, b]}^{(2)}+\left\|\psi_{1}-\bar{\psi}_{1}\right\|_{T}^{(0)}+\left\|\psi_{2}-\bar{\psi}_{2}\right\|_{T}^{(0)}+\|h-\bar{h}\|_{T}^{(1)}\right],
\end{gathered}
$$

is valid, where $c_{6}>0$ depends on the data of problems $A$ and $\bar{A}$ in the set $K_{\alpha}$.

Proof. We construct a function $\tilde{\varphi}(x) \in C^{2+\alpha}[a, b]$ such that

$$
\tilde{\varphi}(x)=\left\{\begin{array}{l}
\varphi(x), \quad x \in\left[\gamma_{1}(0), \gamma_{2}(0)\right], \\
\varphi\left(\gamma_{1}(0)\right), \quad x=\gamma_{1}(t), \quad t \in[0, T] \\
\varphi\left(\gamma_{2}(0)\right), \quad x=\gamma_{2}(t), \quad t \in[0, T]
\end{array}\right.
$$

Consider the function

$$
\begin{aligned}
& F(x, t)=\tilde{\varphi}(x)+\frac{\gamma_{2}(t)-x}{\gamma_{2}(t)-\gamma_{1}(t)}\left[\psi_{1}(t)-\psi_{1}(0)\right]+ \\
& +\frac{x-\gamma_{1}(t)}{\gamma_{2}(t)-\gamma_{1}(t)}\left[\psi_{2}(t)-\psi_{2}(0)\right], \quad(x, t) \in \bar{D} .
\end{aligned}
$$

It's obvious that $F(x, t) \in C^{2+\alpha, 1+\alpha / 2}(\bar{D})$ and $F(x, 0)=\varphi(x), F\left(\gamma_{1}(t), t\right)=$ $\psi_{1}(t), F\left(\gamma_{2}(t), t\right)=\psi_{2}(t)$.

Denote

$$
\begin{gathered}
z(x, t)=u(x, t)-\bar{u}(x, t), \lambda(t)=f(t)-\bar{f}(t), \delta_{1}(x)=g(x)-\bar{g}(x), \\
\delta_{2}(x)=\varphi(x)-\bar{\varphi}(x), \delta_{3}(t)=\psi_{1}(t)-\bar{\psi}_{1}(t), \delta_{4}(t)=\psi_{2}(t)-\bar{\psi}_{2}(t), \\
\delta_{5}(t)=h(t)-\bar{h}(t), \delta_{6}(x, t)=F(x, t)-\bar{F}(x, t), w(x, t)=z(x, t)-\delta_{6}(x, t) .
\end{gathered}
$$

Subtracting from the relations of problem $A$ the corresponding relations of problem $\bar{A}$ we obtain the problem of determining a pair of functions $\{\lambda(t), w(x, t)\}$ :

$$
\begin{gathered}
w_{t}-w_{x x}=\phi(x, t),(x, t) \in D, \\
w(x, 0)=0, \quad x \in\left[\gamma_{1}(0), \gamma_{2}(0)\right], \\
w\left(\gamma_{1}(t), t\right)=w\left(\gamma_{2}(t), t\right)=0, \quad t \in[0, T] \\
\lambda(t)=\left[\delta_{5 t}(t)-\delta_{4}(t) \gamma_{2 t}(t)+\delta_{3}(t) \gamma_{1 t}(t)-z_{x}\left(\gamma_{2}(t), t\right)+z_{x}\left(\gamma_{1}(t), t\right)\right] / \\
/ \int_{\gamma_{1}(t)}^{\gamma_{2}(t)} g(x) d x-H(t) \quad t \in[0, T]
\end{gathered}
$$


where

$$
\begin{gathered}
\phi(x, t)=\lambda(t) g(x)-\bar{f}(t) \delta_{1}(x)+\delta_{6 x x}(x, t)-\delta_{6 t}(x, t), \\
H(t)=\left\{\left[\bar{h}_{t}(t)-\bar{\psi}_{2}(t) \gamma_{2 t}(t)+\bar{\psi}_{1}(t) \gamma_{1 t}(t)-\bar{u}_{x}\left(\gamma_{2}(t), t\right)+\bar{u}_{x}\left(\gamma_{1}(t), t\right)\right] \times\right. \\
\left.\times \int_{\gamma_{1}(t)}^{\gamma_{2}(t)} \delta_{1}(x) d x\right\}\left[\int_{\gamma_{1}(t)}^{\gamma_{2}(t)} g(x) d x \cdot \int_{\gamma_{1}(t)}^{\gamma_{2}(t)} \bar{g}(x) d x\right] .
\end{gathered}
$$

First, we extend $\phi(x, t)$ by setting

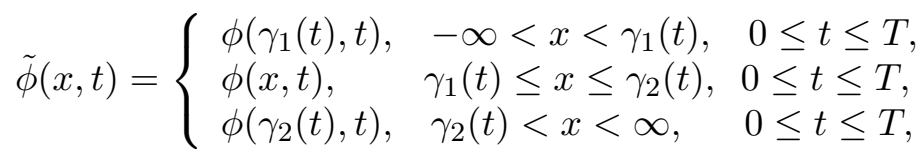

Then the extended $\tilde{\phi}(x, t)$ is bounded continuous and uniformly Hölder continuous for each compact subset of $\bar{D}$.

Consider the potential (see $[1$, chapter 14$]$ )

$$
y(x, t)=\int_{0}^{t} \int_{-\infty}^{+\infty} G(x-\xi, t-\tau) \tilde{\phi}(\xi, \tau) d \xi d \tau,
$$

where

$$
G(x, t)=\frac{1}{\sqrt{4 \pi t}} \exp \left(-\frac{x^{2}}{4 t}\right), t>0
$$

is fundamental solution to equation $y_{t}-y_{x x}=0$.

For $y(x, t)$ the following statements are true

1 ) The function $y(x, t)$ satisfies the equation

$$
y_{t}-y_{x x}=\tilde{\phi}(x, t), \quad(x, t) \in(-\infty,+\infty) \times(0, T]
$$

2) The functions $y(x, t)$ and $y_{x}(x, t)$ are continuous on $(-\infty,+\infty) \times(0, T]$;

$$
\begin{gathered}
\text { 3) }|y(x, t)| \leq c_{7}\|\tilde{\phi}\|_{D}^{(0)} \cdot t, \quad(x, t) \in \bar{D} \\
\text { 4) }\left|y_{x}(x, t)\right| \leq c_{8}\|\tilde{\phi}\|_{D}^{(0)} \cdot t^{1 / 2}, \quad(x, t) \in \bar{D} .
\end{gathered}
$$

The solution to the problem of determining $w(x, t)$ from conditions (2.2), (2.3), (2.4) will be sought in the following form

$$
w(x, t)=v(x, t)+y(x, t)
$$

where $y(x, t)$ is the function known from (2.7), and the function $v(x, t)$ is a solution to the following problem:

$$
\begin{gathered}
v_{t}-v_{x x}=0, \quad(x, t) \in D, \\
v(x, 0)=0, x \in\left[\gamma_{1}(0), \gamma_{2}(0)\right], \\
v\left(\gamma_{1}(t), t\right)=-y\left(\gamma_{1}(t), t\right), v\left(\gamma_{2}(t), t\right)=-y\left(\gamma_{2}(t), t\right), \quad t \in[0, T] .
\end{gathered}
$$


The solution to problem (2.8) can be represented as [3, chapter 14]:

$$
\begin{aligned}
& v(x, t)=\int_{0}^{t} G_{x}\left(x-\gamma_{1}(\tau), t-\tau\right) \rho_{1}(\tau) d \tau+ \\
& +\int_{0}^{t} G_{x}\left(x-\gamma_{2}(\tau), t-\tau\right) \rho_{2}(\tau) d \tau,
\end{aligned}
$$

where $\rho_{1}(t)$ and $\rho_{2}(t)$ are solutions to the system of integral equations:

$$
\begin{aligned}
& \rho_{1}(t)=2 \int_{0}^{t} G_{x}\left(\gamma_{1}(t)-\gamma_{1}(\tau), t-\tau\right) \rho_{1}(\tau) d \tau+ \\
& +2 \int_{0}^{t} G_{x}\left(\gamma_{1}(t)-\gamma_{2}(\tau), t-\tau\right) \rho_{2}(\tau) d \tau+2 y\left(\gamma_{1}(t), t\right), \\
& \rho_{2}(t)=-2 \int_{0}^{t} G_{x}\left(\gamma_{2}(t)-\gamma_{2}(\tau), t-\tau\right) \rho_{2}(\tau) d \tau- \\
& -2 \int_{0}^{t} G_{x}\left(\gamma_{2}(t)-\gamma_{1}(\tau), t-\tau\right) \rho_{1}(\tau) d \tau-2 y\left(\gamma_{2}(t), t\right) .
\end{aligned}
$$

It should be noted that under the conditions of Theorem $2.1 \rho_{1}(t)$, $\rho_{2}(t) \in C[0, T][3$, chapter 14$]$.

Estimate the function $w(x, t)$ a priory

$$
|w(x, t)| \leq|v(x, t)|+|y(x, t)| \quad(x, t) \in D,
$$

For the first term in (2.11) we have:

$$
\begin{gathered}
|v(x, t)| \leq \int_{0}^{t}\left|G_{x}\left(x-\gamma_{1}(\tau), t-\tau\right)\right| \cdot\left|\rho_{1}(\tau)\right| d \tau+ \\
+\int_{0}^{t}\left|G_{x}\left(x-\gamma_{2}(\tau), t-\tau\right)\right| \cdot\left|\rho_{2}(\tau)\right| d \tau .
\end{gathered}
$$

Using the method used in [3, chapter 14] for the derivatives of the fundamental solution, we obtain the estimates

$$
\begin{aligned}
& \left|G_{x}\left(x-\gamma_{i}(\tau), t-\tau\right)\right|,\left|G_{x}\left(\gamma_{i}(t)-\gamma_{i}(\tau), t-\tau\right)\right|, \\
& \mid G_{x}\left(\gamma_{1}(t)-\gamma_{2}(\tau), t-\tau \mid \leq \mathrm{const} \cdot(t-\tau)^{-1 / 2} \quad i=1,2,\right. \\
& \mid G_{x x}\left(\gamma_{i}(t)-\gamma_{i}(\tau), t-\tau|,| G_{x x}\left(\gamma_{1}(t)-\gamma_{2}(\tau), t-\tau \mid \leq\right.\right. \\
& \leq \text { const }(t-\tau)^{-3 / 2}, i=1,2
\end{aligned}
$$

Using estimates (2.12) we obtain

$$
|v(x, t)| \leq c_{9}\left[\left\|\rho_{1}\right\|_{T}^{(0)}+\left\|\rho_{2}\right\|_{T}^{(0)}\right] \cdot T^{1 / 2}
$$


Functions $\rho_{1}(t)$ and $\rho_{2}(t)$ are estimated as follows: from (2.10) we have

$$
\begin{aligned}
& \left|\rho_{1}(t)\right| \leq 2\left|y\left(\gamma_{1}(t), t\right)\right|+2 \int_{0}^{t}\left|G_{x}\left(\gamma_{1}(t)-\gamma_{1}(\tau), t-\tau\right)\right| \cdot\left|\rho_{1}(\tau)\right| d \tau+ \\
& \quad+2 \int_{0}^{t}\left|G_{x}\left(\gamma_{1}(t)-\gamma_{2}(\tau), t-\tau\right)\right| \cdot\left|\rho_{2}(\tau)\right| d \tau, \\
& \left|\rho_{2}(t)\right| \leq 2\left|y\left(\gamma_{2}(t), t\right)\right|+2 \int_{0}^{t}\left|G_{x}\left(\gamma_{2}(t)-\gamma_{2}(\tau), t-\tau\right)\right| \cdot\left|\rho_{2}(\tau)\right| d \tau+ \\
& +2 \int_{0}^{t}\left|G_{x}\left(\gamma_{2}(t)-\gamma_{1}(\tau), t-\tau\right)\right| \cdot\left|\rho_{1}(\tau)\right| d \tau, \quad t \in[0, T] .
\end{aligned}
$$

For the right-hand sides of (2.14) and the inequality after (2.14), by virtue of the estimates for $|y(\cdot)|$ and (2.12), we can write that

$$
\begin{aligned}
& \left|\rho_{1}(t)\right| \leq c_{9}\|\tilde{\phi}\|_{D}^{(0)} \cdot t+c_{10} \cdot t^{1 / 2} \rho, \\
& \left|\rho_{2}(t)\right| \leq c_{11}\|\tilde{\phi}\|_{D}^{(0)} \cdot t+c_{12} \cdot t^{1 / 2} \rho,
\end{aligned}
$$

where $c_{9}, c_{10}, c_{11}, c_{12}$ are positive constants, $\rho=\left\|\rho_{1}\right\|_{T}^{(0)}+\left\|\rho_{2}\right\|_{T}^{(0)}$. The leff-hand sides of the last inequalities hold for any $t \in[0, T]$, then we can write that

or

$$
\begin{gathered}
\left\|\rho_{1}(t)\right\|_{T}^{(0)} \leq c_{9}\|\tilde{\phi}\|_{D}^{(0)} \cdot T+c_{10} \cdot T^{1 / 2} \rho, \\
\left\|\rho_{1}(t)\right\|_{T}^{(0)} \leq c_{11}\|\tilde{\phi}\|_{D}^{(0)} \cdot T+c_{12} \cdot T^{1 / 2} \rho,
\end{gathered}
$$

$$
\rho \leq c_{13}\|\tilde{\phi}\|_{D}^{(0)} \cdot T+c_{14} \cdot T^{1 / 2} \rho,
$$

where $c_{13}, c_{14}>0$ depend on the data of problems $\bar{A}$ and $A$ on the set $K_{\alpha}$.

Let $0<T_{1} \leq T$ be a number such that $c_{14} T_{1}^{1 / 2}<1$. Then for all $t \in\left[0, T_{1}\right]$ we have

$$
\left\|\rho_{1}\right\|_{T_{1}}^{(0)}+\left\|\rho_{2}\right\|_{T_{1}}^{(0)} \leq c_{15}\|\tilde{\phi}\|_{D}^{(0)} \cdot T_{1} .
$$

Considering (2.15) in (2.13) we get:

$$
|v(x, t)| \leq c_{16}\|\tilde{\phi}\|_{T}^{(0)} \cdot T_{1}^{3 / 2}, \quad(x, t) \in\left[\gamma_{1}(t), \gamma_{2}(t)\right] \times\left[0, T_{1}\right]
$$

Taking into account estimate $|y(x, t)| \leq$ const $\|\tilde{\phi}\|_{D}^{(0)} \cdot T$ for $|w(x, t)|$, we obtain from (2.11) that

$$
|w(x, t)| \leq c_{17}\|\tilde{\phi}\|_{D}^{(0)} \cdot T_{1}, \quad(x, t) \in\left[\gamma_{1}(t), \gamma_{2}(t)\right] \times\left[0, T_{1}\right] .
$$

From here for $z(x, t)=w(x, t)+\delta_{6}(x, t)$ one can write

$$
|z(x, t)| \leq c_{17}\|\tilde{\phi}\|_{D}^{(0)} \cdot T_{1}+\left\|\delta_{6}\right\|_{D}^{(2,1)}
$$


or

$$
|z(x, t)| \leq c_{18}\left[\left\|\delta_{1}\right\|_{[a, b]}^{(0)}+\left\|\delta_{6}\right\|_{D}^{(2,1)}\right]+c_{19} \theta T_{1}, \quad(x, t) \in D
$$

where $c_{18}, c_{19}>0$ depend on the data of problems $A, \bar{A}$ and on the set $K_{\alpha}$

$$
\theta=\|\lambda\|_{T}^{(0)}+\|z\|_{D}^{(0)} .
$$

Let us estimate the function $\lambda(t)$. From (2.5) we have

$$
\begin{gathered}
|\lambda(t)| \leq\left[\left|\delta_{5 t}(t)\right|+\left|\delta_{4}(t)\right|\left|\gamma_{2 t}(t)\right|+\left|\delta_{3}(t)\right|\left|\gamma_{1 t}(t)\right|+\right. \\
\left.+\left|z_{x}\left(\gamma_{1}(t), t\right)\right|+\mid z_{x}\left(\gamma_{2}(t), t\right)\right] / \int_{\gamma_{1}(t)}^{\gamma_{2}(t)}|g(x)| d x+|H(t)| .
\end{gathered}
$$

Taking into account the conditions of the theorem and (2.12), for $z_{x}\left(\gamma_{1}(t), t\right)$ we obtain

$$
\begin{gathered}
\left.\left|z_{x}\left(\gamma_{1}(t), t\right)\right| \leq\left|v_{x}\left(\gamma_{1}(t), t\right)\right|+\left|y_{x}\left(\gamma_{1}(t), t\right)\right|+\left|\delta_{6 x}\left(\gamma_{1}(t), t\right)\right|\right] \leq \\
\leq\left\|\delta_{6}\right\|_{D}^{(1,0)}+c_{20}\|\tilde{\phi}\|_{D}^{(1,0)} \cdot T^{1 / 2}+\int_{0}^{t}\left|G_{x x}\left(\gamma_{1}(t)-\gamma_{1}(\tau), t-\tau\right)\right|\left|\rho_{1}(\tau)\right| d \tau+ \\
+\int_{0}^{t}\left|G_{x x}\left(\gamma_{1}(t)-\gamma_{2}(\tau), t-\tau\right)\right|\left|\rho_{2}(\tau)\right| d \tau
\end{gathered}
$$

or

$$
\left|z_{x}\left(\gamma_{1}(t), t\right)\right| \leq c_{21}\left[\left\|\delta_{1}\right\|_{[a, b]}^{(0)}+\left\|\delta_{6}\right\|_{D}^{(2,1)}\right]+c_{22} \theta T^{1 / 2},
$$

where $c_{21}, c_{22}>0$ depend on the data of problems $A, \tilde{A}$ and on the set $K_{\alpha}$. For $\left|z_{x}\left(\gamma_{2}(t), t\right)\right|$ a similar estimate is obtained.

Thus, for $|\lambda(t)|$ we have

$$
|\lambda(t)| \leq c_{23}\left[\left\|\delta_{1}\right\|_{[a, b]}^{(0)}+\left\|\delta_{5}\right\|_{T}^{(1)}+\left\|\delta_{6}\right\|_{D}^{(2,1)}\right]+c_{24} \theta T^{1 / 2}, \quad t \in[0, T],
$$

Estimates (2.16) and (2.19) are satisfied for any values of $(x, t) \in\left[\gamma_{1}(t), \gamma_{2}(t)\right] \times$ $\left[0, T_{1}\right]$, then for them we can assert

$$
\begin{gathered}
\|z\|_{D}^{(0)} \leq c_{18}\left[\left\|\delta_{1}\right\|_{[a, b]}^{(0)}+\left\|\delta_{6}\right\|_{D}^{(2,1)}\right]+c_{19} \theta T^{1 / 2} \\
\|\lambda(t)\|_{T}^{(0)} \leq c_{23}\left[\left\|\delta_{1}\right\|_{[a, b]}^{(0)}+\left\|\delta_{6}\right\|_{D}^{(2,1)}+\left\|\delta_{5}\right\|_{T}^{(1)}\right]+c_{24} \theta T^{1 / 2} .
\end{gathered}
$$

Adding the last two inequalities, we obtain

$$
\theta \leq c_{25}\left[\left\|\delta_{1}\right\|_{[a, b]}^{(0)}+\left\|\delta_{6}\right\|_{D}^{(2,1)}\right]+c_{26} \theta T^{1 / 2}
$$

where $c_{25}, c_{26}>0$ depend on the data of problems $A, \tilde{A}$ and on the set $K_{\alpha}$.

Let $0<T_{2} \leq T$, be a number such that $c_{26} T_{2}^{1 / 2}<1$. Then from the last inequality we obtain an estimate of the "conditional" stability of the solution for $(x, t) \in\left[\gamma_{1}(t), \gamma_{2}(t)\right] \times\left[0, T^{*}\right], T^{*}=\min \left\{T_{1}, T_{2}\right\}$

$$
\|\lambda\|_{T}^{(0)}+\|z\|_{D}^{(2,1)} \leq c_{27}\left[\left\|\delta_{1}\right\|_{[a, b]}^{(0)}+\left\|\delta_{2}\right\|_{\left[\gamma_{1}(0), \gamma_{2}(0)\right]}^{(2)}+\left\|\delta_{3}\right\|_{T}^{(1)}+\left\|\delta_{4}\right\|_{T}^{(1)}+\left\|\delta_{5}\right\|_{T}^{(1)}\right] .
$$


The uniqueness of the solution to problem (1.1)-(1.4) is obtained from the last inequality for

$$
g(x) \equiv \bar{g}(x), \varphi(x) \equiv \bar{\varphi}(x), \psi_{1}(t) \equiv \bar{\psi}_{1}(t), \psi_{2}(t) \equiv \bar{\psi}_{2}(t), h(t) \equiv \bar{h}(t) .
$$

The theorem has been proved.

\section{References}

[1] A.Ya. Akhundov, Inverse problems for quasilinear parabolic equations in domains with a moving boundary, Preprint, Baku, 2000, 18 pp.

[2] A.Ya. Akhundov, Inverse problems for linear and quasilinear parabolic equations, Dissertation abstract for the degree of Doctor of physical and mathematical sciences, Baku, 2008, 35 pp.

[3] J.R. Cannon, The one-dimensional heat equation, Cambridge, Cambridge University Press \& Reading, MA, Addison Wesley XXV (1984), 483 pp.

[4] E.M. Kartashov, New integral relations for analytical solutions of parabolic equations in noncylindrical domains, Reports of the Academy of Sciences 374, 2000, no.2, 168-172. (Russian)

[5] O.A. Ladyzhenskaya, V.A. Solonnikov, N.N. Uraltseva, Linear and quasilinear equations of parabolic type, M. Nauka, 1967, 736 pp. (Russian)

[6] A.N. Tikhonov, A.A. Samarskiy, Equations of mathematical physics, Moscow, Nauka, 1966, $724 \mathrm{pp}$.

Adalat Ya. Akhundov

Institute of Mathematics and Mechanics of NAS of Azerbaijan, Baku, Azerbaijan

E-mail address: adalatakhund@mail.ru

Arasta Sh. Habibova

Lankaran State University, Lankaran, Azerbaijan

E-mail address: arasta.h@mail.ru

Received: January 1, 2021; Revised: April 12, 2021; Accepted: June 3, 2021 